\title{
Valvoplastia mitral em pacientes jovens com cardiopatia reumática
}

Fernando Antônio FANTINI*, Leonardo Ferber DRUMOND**, Bayard GONTIJO FILHO*, Mário Osvaldo VRANDECIC**, Joāo Alfredo de PAULA E SILVA*, Juscelino Teixeira BARBOSA*, Luiz Fernando

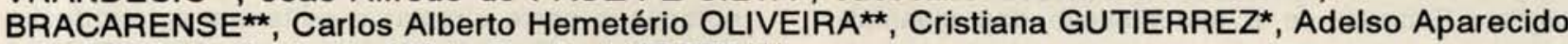

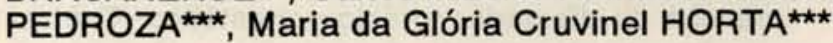

RBCCV 44205-165

FANTINI, F.A.; DRUMOND, L. F.; GONTIJO FILHO, B.; VRANDECIC, M. O.; PAULA E SILVA, J. A.; BARBOSA, J. T.; BRACARENSE, L. F.; OLIVEIRA, C. A. H.; GUTIERREZ, C.; PEDROZA, A. A.; HORTA, M. G. C. - Valvoplastia mitral em pacientes jovens com cardiopatia reumática. Rev. Bras. Cir. Cardiovasc., 7(2):78-87,1992.

RESUMO: No período, de setembro de 1988 a janeiro de 1992, 56 pacientes com até 20 anos de idade ( 4 a 20 anos, média de 12,7) com insuficiência mitral pura ou predominante de etiologia reumática foram submetidos a valvoplastia mitral. Noventa e quatro por cento dos pacientes estavam em classe funcional III ou IV da NYHA. A técnica cirúrgica básica usada em todos os pacientes foi a anuloplastia assimétrica preconizada por Reed et alii ${ }^{31}$, associada em $69,7 \%$ dos casos a outros procedimentos sobre as cúspides e aparelho subvalvar mitral. Dois pacientes foram submetidos, concomitantemente, a plastia da valva tricúspide e 4 a troca da valva aórtica. Estudo ecodopplercardiográfico per-operatório foi utilizado após a correção em todos os casos e mostrou ausência de lesōes residuais em $76 \%$ dos pacientes e insuficiência mitral discreta nos demais. Não houve mortalidade hospitalar. Ecopplercardiograma realizado antes da alta hospitalar mostrou boa correlação com o estudo per-operatório. Foi possível colher informaçōes do seguimento tardio de 53 pacientes. Ocorreu 1 óbito tardio três meses após a cirurgia, por morte súbita. Quatro pacientes foram reoperados e submetidos a troca valvar: uma paciente no $4^{\circ}$ mês de pós-operatório (PO), por falha primária do procedimento, e outros três no $6^{\circ}, 34^{\circ}$ e $38^{\circ}$ meses de $\mathrm{PO}$, por comprovada recidiva da cardite reumática. Os demais encontram-se em classe funcional I e II (NYHA). Concluímos, baseados nos resultados apresentados, que a anuloplastia mitral assimétrica é um excelente procedimento para pacientes jovens com valvopatia reumática, constituindo-se numa boa alternativa à troca de valva ou implante de anéis, sendo, no entanto, extremamente importante o controle de recidivas da doença reumática. reumática.

DESCRITORES: valva mitral, cirurgia; cardiopatia reumática, valvoplastia mitral; valva mitral, cardiopatia

\section{INTRODUÇĀO}

As complicaçōes causadas pelo implante de próteses valvulares têm motivado grande interesse pela preservação das valvas nativas, através de diversas técnicas de valvoplastia. Além disso, a alta prevalência da doença reumática em nosso meio, principalmente na populaçāo menos favorecida, im- pōe, muitas vezes, uma indicação cirúrgica mais precoce, em pacientes mais sujeitos a atividade reumática recorrente ou persistente.

Diante desses fatos, iniciamos, em 1988, um programa de tratamento cirúrgico conservador da valva mitral, em pacientes jovens portadores de insuficiência valvar reumática, que foram submetidos

\footnotetext{
Trabalho realizado no Biocor Hospital de Doenças Cardiovasculares e na Santa Casa de Misericórdia de Belo Horizonte, MG, Brasil. Apresentado ao $19^{\circ}$ Congresso Nacional de Cirurgia Cardiaca. São Paulo, SP, 7 a 9 de maio, 1992.

Laureado com o Prêmio Nacional de Cirurgia Cardiaca.

* Do Biocor Hospital e da Santa Casa de Misericórdia.

* Do Biocor Hospital.

** Da Santa Casa de Misericórdia.

Endereço para separatas: Fernando A. Fantini. Av. Carandaí, 78, Apto, 103. 30130 Belo Horizonte, MG, Brasil.
} 
FANTINI, F.A.; DRUMOND, L. F.; GONTIJO FILHO, B.; VRANDECIC, M. O.; PAULA E SILVA, J. A.; BARBOSA, J. T.; BRACARENSE, L. F.; OLIVEIRA, C. A. H.; GUTIERREZ, C.; PEDROZA, A. A.; HORTA, M. G. C. - Valvoplastia mitral em pacientes jovens com cardiopatia reumática. Rev. Bras. Cir. Cardiovasc., 7(2):78-87,1992.

a uma técnica de valvoplastia estandardizada. Os resultados obtidos são apresentados no presente trabalho.

\section{CASUÍSTICA E MÉTODOS}

No período compreendido entre setembro de 1988 e janeiro de 1992, 56 pacientes com idade variando de 4 a 20 anos (média: $12,7 \pm 5,12$ ), portadores de insuficiência mitral pura ou predominante de etiologia reumática, foram submetidos a valvoplastia mitral. Quarenta $(71 \%)$ pacientes eram do sexo feminino e 16 do masculino. 0 Gráfico 1 mostra a distribuição dos pacientes de acordo com idade e sexo.

\section{GRÁFICO 1 \\ DISTRIBUIÇĀO DOS PACIENTES DE ACORDO COM O SEXO E IDADE}

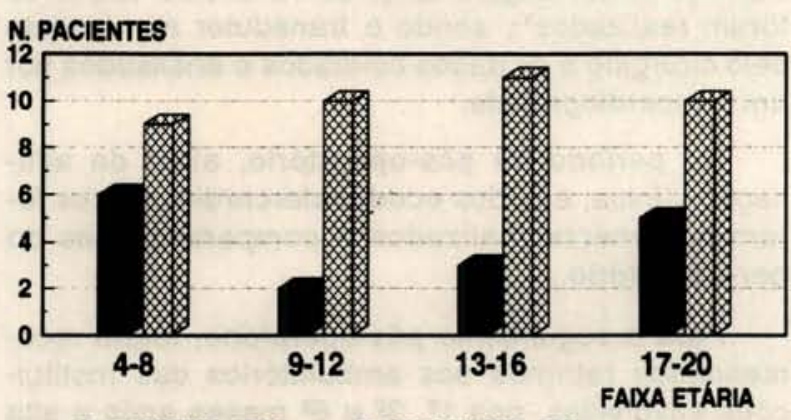

$\otimes$ FEMININO: $71 \%$

MASCULINO: $29 \%$

No pré-operatório, $53(94,65)$ estavam em classe funcional III ou IV da New Heart Association (NYHA). A cirurgia foi indicada em 3 pacientes em classe funcional II, que apresentavam insuficiência mitral severa, com aumento acentuado das dimensōes do átrio esquerdo e aumento dos índices do volume sistólico final acima de $60 \mathrm{ml} / \mathrm{m}^{2}$ e diastólico final acima de $160 \mathrm{ml} / \mathrm{m}^{2}$. Quatro $(7,14 \%)$ pacientes apresentavam fibrilação atrial. Foi necessário indicar-se a cirurgia em 4 (7,14\%) que se encontravam na fase aguda da cardite reumática e grave instabilidade hemodinâmica. O diagnóstico etiológico foi baseado em dados clínicos, laboratoriais, aspectos ecocardiográfico e cirúrgico e, em alguns casos, em exames anatomopatológicos. Os dados clínicos de maior relevância estão relacionados na Tabela 1.

O diagnóstico clínico foi confirmado em todos os casos através de estudos ecodopplercardiográficos, sendo que os critérios básicos de seleção dos pacientes para o procedimento foram baseados nesse exame complementar. Foram cuidadosamen-
TABELA 1

DADOS CLÍNICOS
56

SET./88 A JAN./92

4 a 20 anos
$12,7+5,12$
$3(5,4 \%)$
$28(50,0 \%)$
$25(44,6 \%)$
$4(7,14 \%)$
$4(7,14 \%)$

te observados a textura e a mobilidade das cúspides, estado do aparelho subvalvar, presença e grau de fusão comissural, presença e grau de dilatação do anel valvar $e$ índices de função sistólica e distólica do ventrículo esquerdo. Não foram considerados para o procedimento pacientes com espessamento e retraçāo importante da cúspide anterior, acometimento extenso do aparelho subvalvar, fusāo comissural de grau importante e calcificação extensa dos diversos componentes do aparelho valvar. No entanto, foi fundamental a análise da valva pelo cirurgiāo no per-operatório. Assim, durante 0 ato cirurgico, 3 pacientes selecionados para o procedimento, com base nos critérios acima, foram considerados inadequados, sendo submetidos a troca valvar. Por outro lado, em uma criança encaminhada para troca valvar pela presença de alteraçōes importantes do aparelho subvalvar com retração de cordas e fusāo papilovalvar foi possível a realizaçāo da técnica, com bom resultado funcional. As lesōes valvares mitrais encontradas, bem como as lesōes associadas, sāo mostradas na Tabela 2.

Todos os pacientes foram operados através de esternotomia mediana, hipotermia sistêmica moderada e cardioplegia cristalóide. Em alguns pacientes portadores de insuficiência aórtica, utilizamos infu-

TABELA 2

LESÕES VALVARES: DIAGNÓSTICO ECOCARDIOGRÁFICO

\begin{tabular}{lcr}
\hline & N & $\%$ \\
\hline INSUFICIÊNCIA MITRAL PURA & 37 & 66,1 \\
DUPLA LESÁO MITRAL & 19 & 33,9 \\
INSUFICIÊNCIA AÓRTICA LEVE & 14 & 25,0 \\
INSUFICIÊNCIA AÓRTICA SEVERA & 3 & 5,4 \\
DUPLA LESÁO AÓRTICA & 1 & 1,8 \\
INSUFICIEENCIA TRICÚSPIDE & 8 & 14,3 \\
LEVE/MODERADA & 8 & \\
INSUFICIÊNCIA TRICÚSPIDE & 2 & 3,6 \\
MODERADA/SEVERA & 2 &
\end{tabular}


FANTINI, F.A.; DRUMOND, L. F.; GONTIJO FILHO, B.; VRANDECIC, M. O.; PAULA E SILVA, J. A.; BARBOSA, J. T.; BRACARENSE, L. F.; OLIVEIRA, C. A. H.; GUTIERREZ, C.; PEDROZA, A. A.; HORTA, M. G. C. - Valvoplastia mitral em pacientes jovens com cardiopatia reumática. Rev. Bras. Cir. Cardiovasc., 7(2):78-87,1992.

são da solução cardioplégica, retrogradamente pelo seio coronário, ou diretamente nos óstios coronários. $O$ átrio esquerdo foi aberto anteriormente às veias pulmonares direitas $e$, em geral, propiciou uma exposição adequada do aparelho valvar mitral, visto que a maioria dos pacientes apresentava aumento significativo da cavidade. A técnica cirúrgica básica utilizada em todos os casos foi a anuloplastia mitral assimétrica descrita por REED et alii ${ }^{31}$. Em cerca de $30 \%$ dos casos, essa foi a única técnica empregada. No entanto, nos demais pacientes, outros procedimentos sobre as cúspides e aparelho subvalvar foram necessários (Tabela 3 ).

TABELA 3

TÉCNICA CIRÚRGICA

\begin{tabular}{lrr}
\hline & $\mathrm{N}^{\bullet}$ & $\%$ \\
\hline ANULOPLASTIA ISOLADA & 17 & 30,4 \\
ANULOPLASTIA ASSOCIADA & 39 & 69,6 \\
Encurtamento cordas cúspide anterior & 17 & 30,4 \\
Ressecçāo cordas rotas cúspide posterior* & 3 & 5,4 \\
Ressecçāo cordas cúspide posterior & 3 & 5,4 \\
Correçāo retraçāo cúspide posterior & 14 & 25,0 \\
Sutura & 4 & 7,1 \\
Ressecçāo* & 10 & 17,8 \\
Comissurotomia & 7 & 12,5 \\
Papilarotomia & 2 & 3,6 \\
Fenestraçāo de cordas & 1 & 1,8 \\
\hline
\end{tabular}

* Ressecção quadrangular.

Deste modo, foi realizado encurtamento de cordas da cúspide anterior em 17 casos, ressecção de cordas da cúspide posterior em 3 e ressecção quadrangular na cúspide posterior, devido à presença de rotas em outros 3 pacientes. A presença de retraçōes localizadas na cúspide posterior, principalmente ao nível da implantação das cordas primárias, levando à formação de defeitos semelhantes a pequenas fendas, foi corrigida em 4 casos através da aplicação de pontos separados de Prolene 5-0. Porém, em outros 10 casos, foi necessária a ressecção da parte retraída ou espessada da cúspide, o que facilitou a sua coaptaçāo. Pequenas comissurotomias foram feitas em 7 pacientes. Em todos os casos, uma faixa de pericárdio bovino preservado em glutaraldeído foi suturada ao longo da metade posterior do anel, tanto para ancorar os pontos anulares, como para evitar uma posterior redilatação do anel valvar. Após a correção, foram realizados testes de suficiência através do enchimento do ventrículo esquerdo com jatos sob pressão de solução salina.

Quatro pacientes foram submetidos, concomitantemente, a troca de válva aórtica e 2 outros
TABELA 4

PROCEDIMENTOS ASSOCIADOS

\begin{tabular}{lcc}
\hline & $\mathrm{N}^{2}$ & $\%$ \\
\hline TROCA DE VALVA AÓRTICA & 4 & 7,1 \\
$\begin{array}{l}\text { PLASTIA DE VALVA TRICÚSPIDE } \\
\text { (DEVEGA) }\end{array}$ & 2 & 3,6 \\
\hline
\end{tabular}

a plastia da valva tricúspide pela técnica de De Vega (Tabela 4).

Após a saída de circulação extracorpórea, todos os pacientes foram submetidos a exame ecodopplercardiográfico intra-operatório pelo método epicárdico, para avaliação do resultado da operação. Com o paciente heparinizado e a cânula aórtica em posição, as cânulas atriais foram retiradas $e$, após completa estabilização hemodinâmica, estudos bidimensionais de Doppler nos cortes modificados nos eixos longo e curto do ventrículo esquerdo foram realizados ${ }^{13}$, sendo 0 transdutor manipulado pelo cirurgiāo e os dados coletados e analisados por um ecocardiografista.

No período de pós-operatório, além da avaliaçāo clínica, estudos ecodopplercardiográficos foram novamente realizados e comparados aos do per-operatório.

Para o seguimento pós-operatórlo, foram recomendados retornos aos ambulatórios das Instituiçōes envolvidas, nos $1^{2}, 3^{2}$ e $6^{2}$ meses após a alta e, em seguida, semestralmente, o que não foi possível, em parte pela presença de pacientes de cidades distantes e de baixo poder aquisitivo. Assim, informaçōes de alguns pacientes só foram possíveis através de contato com o médico assistente.

A freqüência de óbitos tardios e complicaçōes que levaram a reoperação foram analisados pelas curvas atuariais obtidas pelo método da tabela de vida e taxas linearizadas de risco. Foram obtidas curvas atuarias de sobrevivência, livre de reoperaçāo e livre de eventos relacionados à valva, com intervalo de confiança de $68 \%$ calculada pela taxa de erro-padrâo. Os intervalos de outros parâmetros foram calculados com base no desvio-padrão.

\section{RESULTADOS}

O período de circulação extracorpórea variou de 40 a 125 minutos (média $77,4 \pm 20,7$ ). O período de clampeamento aórtico variou de 19 a 100 minutos (média $56,7 \pm 19,0$ ), tendo sido de $53,3 \pm 16,6$ minutos nos casos sem procedimentos associados e de $84,5 \pm 15,1$ nos pacientes submetidos, concomitantemente, a troca de valva aórtica ou plastia da 
FANTINI, F.A.; DRUMOND, L. F.; GONTIJO FILHO, B.; VRANDECIC, M. O.; PAULA E SILVA, J. A.; BARBOSA, J. T.; BRACARENSE, L. F.; OLIVEIRA, C. A. H.; GUTIERREZ, C.; PEDROZA, A. A.; HORTA, M. G. C. - Valvoplastia mitral em pacientes jovens com cardiopatia reumática. Rev. Bras. Cir. Cardiovasc., 7(2):78-87,1992.

tricúspide. O estudo ecodopplercardiográfico préoperatório mostrou ausência de lesōes residuais em $76 \%$ dos pacientes e insuficiência mitral discreta nos demais.

Nāo houve mortalidade hospitalar. A evoluçāo pós-operatória, bem como as complicaçōes que ocorreram nesse período podem ser observadas na Tabela 5 .

TABELA 5 EVOLUÇĀO PÓS-OPERATÓRIA

\begin{tabular}{lrr}
\hline & N & $\%$ \\
\hline AUSÊNCIA DE COMPLICAÇŌES & 34 & 60,7 \\
INSUFICIENNCIA CARDIACCA & 10 & 17,8 \\
SINDROME DE BAIXO DÉBITO & 6 & 10,7 \\
INFECÇĀO PULMONAR & 2 & 3,6 \\
SANGRAMENTO PÓS-OPERATÓRIO & & \\
IMEDIATO & 2 & 3,6 \\
OBSTRUÇĀO RESPIRATÓRIA ALTA & 1 & 1,8 \\
HEMOTÓRAX DIREITO* & 1 & 1,8 \\
\hline
\end{tabular}

* Acidente de punçáo de veia subclávia

O estudo ecodopplercardiográfico realizado no pós-operatório mostrou uma correlaçāo de $87,5 \%$ em relaçāo ao exame per-operatório. Nos demais, a insuficiência mitral observada no exame intraoperatório foi subestimada em um grau. Assim, por ocasiāo da alta, $64,3 \%$ dos pacientes apresentavam ausência de insuficiência mitral, em $32,1 \%$ esta era leve e em $3,6 \%$ moderada. A Tabela 6 mostra a correlaçāo entre o ecocardiograma per e pós-operatório.

Foi possível colher informaçōes adequadas e consistentes do seguimento tardio de $53(94,6 \%)$, o que corresponde a 863,8 pacientes/mês (ou 72,0 pacientes/ano), por um período que variou de 1 a 40 meses (média 16,3). Ocorreu um óbito tardio $(1,8 \% \pm 13,4 \%)$, por morte súbita no $3^{2}$ mês após a alta hospitalar, numa paciente de 17 anos que havia sido avaliada ambulatorialmente alguns dias antes e cujo estudo ecocardiográfico mostrava

TABELA 6

ECOCARDIOGRAMA PER E PÓS-OPERATÓRIO

\begin{tabular}{lccccc}
\hline & \multicolumn{2}{c}{ PER } & \multicolumn{2}{c}{ PÓS } \\
\hline INSUFICIÊNCIA & & & & \\
MITRAL & $N^{\circ}$ & $\%$ & $N^{\circ}$ & $\%$ \\
\hline AUSENTE & 43 & 76,8 & 36 & 64,3 \\
LEVE & 13 & 23,2 & 18 & 32,1 \\
MODERADA & 0 & 0 & 2 & 3,6 \\
\hline
\end{tabular}

ausência de lesões residuais com boa função ventricular. Não foi possível a realização de necropsia. Isto corresponde a uma taxa de risco linearizada para óbito de 1,39\% por paciente/ano. A curva atuarial de sobrevida é mostrada no Gráfico 2 e revela que $98,1 \% \pm 1,95 \%$ dos pacientes encontravam-se vivos ao final do período estudado.

\section{GRÁFICO 2}

CURVA ATUARIAL DE SOBREVIDA DE PACIENTES SUBMETIDOS A PLASTIA MITRAL

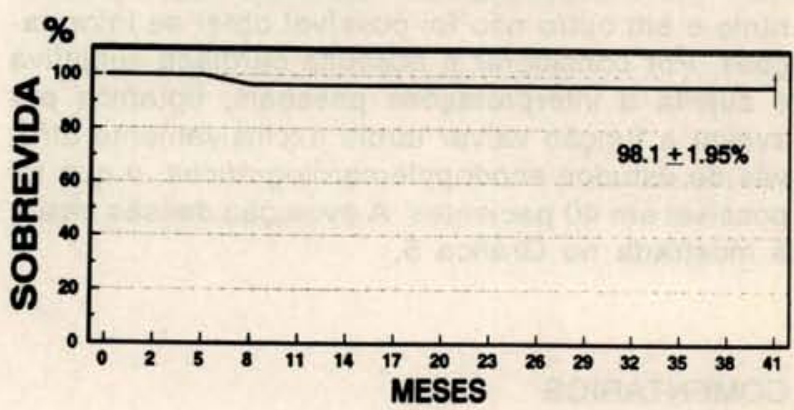

Näo foram observados casos de endocardite ou de embolia arterial, embora esta possa ter sido a causa do óbito observado, face às circunstâncias em que ocorreu.

Quatro pacientes foram reoperados $(7,14 \% \pm$ $25,8 \%$ ) e submetidos a troca da valva mitral, sem mortalidade no período hospitalar, Uma paciente de 12 anos retornou no $4^{2}$ mês de pós-operatório com quadro de insuficiência cardíaca (NYHA III) e insuficiência mitral severa, após ter recebido alta sem lesōes residuais. A reoperaçāo mostrou rotura de um dos pontos anulares, caracterizando falha primária do procedimento. Outros três pacientes tiveram comprovada recidiva da doença reumática. Um paciente de 14 anos, operado na vigência de cardite reumática, foi reoperado no $6^{2}$ mês de pós-operatório. Apresentava insuficiência mitral severa e insuficiência aórtica leve, ausente na primeira internaçāo, tendo relatado que nảo fez uso da medicaçāo prescrita após a alta (penicilina, corticósteróides e medicação anticongestiva). A reoperaçāo demonstrou que a correçāo encontravase intacta, porém havia uma retraçāo acentuada das cúspides, principalmente da anterior. O exame anatomopatológico da peça mostrou sinais de inflamação aguda, caracterizando doença reumática em atividade. Os outros 2 pacientes foram reoperados nos $34^{\circ}$ e $38^{\circ}$ meses de pós-operatório. Ambos tiveram internaçōes para tratamento de cardite reumática no nosso Serviço, evoluíram com intolerância progressiva aos exercícios e as reoperaçōes 
FANTINI, F.A.; DRUMOND, L. F.; GONTIJO FILHO, B.; VRANDECIC, M. O.; PAULA E SILVA, J. A.; BARBOSA, J. T.; BRACARENSE, L. F.; OLIVEIRA, C. A. H.; GUTIERREZ, C.; PEDROZA, A. A.; HORTA, M. G. C. - Valvoplastia mitral em pacientes jovens com cardiopatia reumática. Rev. Bras. Cir. Cardiovasc., 7(2):78-87,1992.

foram indicadas devido a estenose mitral severa, com ausência de insuficiência. Assim, 92,4\% $\pm 3,68 \%$ dos pacientes encontravam-se livres de reoperaçōes, como pode ser visto no Gráfico 3, para uma taxa de risco linearizada de $5,56 \%$ por paciente/ano. $O$ Gráfico 4 mostra que $90,5 \% \pm 3,3 \%$ dos pacientes estavam livres de quaisquer eventos relacionados à valva, sendo a taxa de risco linearizada de $6,95 \%$.

Os demais 47 pacientes em seguimento encontram-se em classe funcional I e II da NYHA. Dos pacientes em fibrilação atrial no pré-operatório, 1 retornou a ritmo sinusal, 2 continuam com o mesmo ritmo e em outro nāo foi possível obter-se informaçōes. Por considerar a ausculta cardíaca subjetiva e sujeita a interpretaçōes pessoais, optamos por avaliar a funçāo valvar tardia exclusivamente através de estudos ecodopplercardiográficos, o que foi possível em 40 pacientes. A evoluçāo desses casos é mostrada no Gráfico 5 .

\section{COMENTÁRIOS}

O melhor nível sócio-econômico e cultural, aliado a uma profilaxia adequada ${ }^{35}$, tem, praticamente, levado à erradicação da doença reumá-

\section{GRÁFICO 3}

CURVA ATUARIAL DE PACIENTES LIVRES DE REOPERAÇĀO APÓS PLASTIA MITRAL

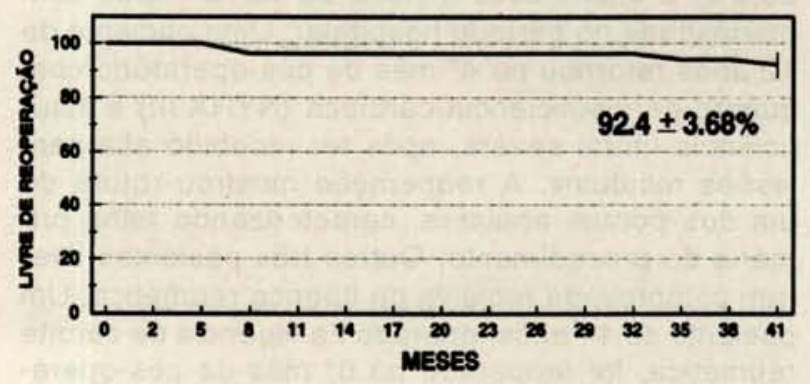

GRÁFICO 4

CURVA ATUARIAL DE PACIENTES LIVRES DE EVENTOS

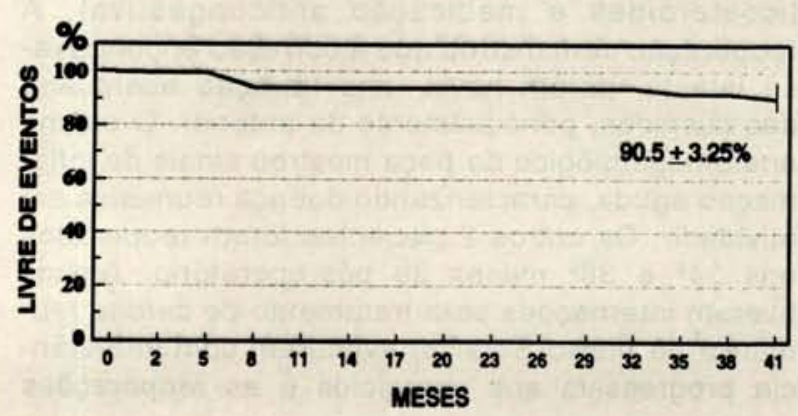

\section{GRÁFICO 5}

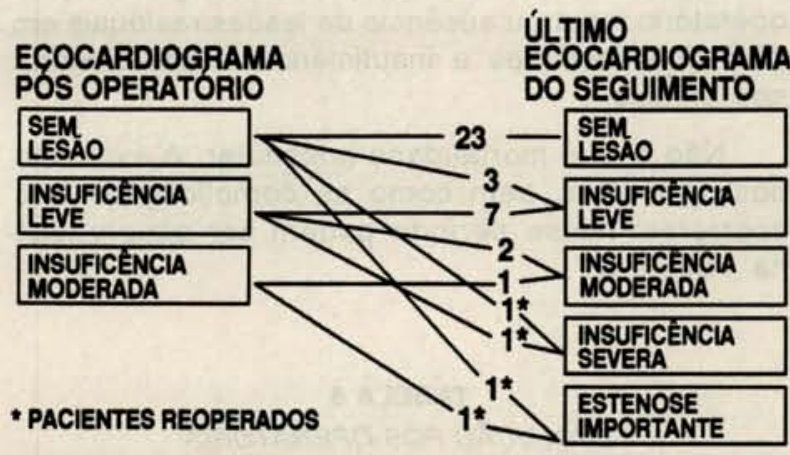

EVOLUÇÁO ECOCARDIOGRÁFICA DE 40 PACIENTES EM SEGUIMENTO. NOTE-SE QUE EM $77.5 \%$ DOS PACIENTES NÁO HOUVE PROGRESSÁO DE LESÓES RESIDUAIS.

tica nos países desenvolvidos. Porém, nos países em desenvolvimento, esta enfermidade mantém um caráter endêmico, atingindo mais de $20 \mathrm{mi}$ Ihōes de pessoas por ano ${ }^{16} \mathrm{e}$ acarretando lesōes valvares cardíacas graves em crianças e adultos jovens ${ }^{2,24}$.

Nos pacientes com indicaçōes cirúrgica, as razōes que têm motivado um maior interesse pela preservação da valva nativa, tanto no nosso meio 9, 10, 20, 24, 28, quanto em outros países 2. 8. 21, 29, 34 têm sido: 1) mau desempenho das próteses artificiais em posição mitral, principalmente no grupo de pacientes jovens; 2) menor mortalidade tanto precoce quanto tardia; 3) melhor desempenho ventricular no pós-operatório; 4) menores índices de endocardite e trombo-embolismo, o que torna desnecessário o uso de anticoagulação prolongada.

As complicaçōes com o uso de próteses artificiais mecânicas e biológicas já são bastante conhecidas e não serão aqui discutidas.

Quanto à mortalidade, estudos comparando grupos submetidos a plastia e troca valvar têm demonstrado melhor resultado com a primeira. COSGROVE \& STEWART ${ }^{15}$ publicaram, em 1989 , a experiência cumulativa da literatura mundial comparando os dois procedimentos, mostrando ser a diferença entre os resultados estatisticamente significativa. Esse fato foi também observado na presente série, já que não ocorreu mortalidade hospitalar.

Tem-se demonstrado que a preservação da continuidade entre $o$ anel mitral e a parede do ventrículo esquerdo através das cordas tendíneas e músculos papilares, desempenha importante papel na funçāo ventricular após a correção da insuficiência mitral crônica. Estudos experimentais, como os desenvolvidos por SARRIS et alii ${ }^{33} \mathrm{e}$ HANSEN et alii ${ }^{26}$, assim como trabalhos comparando os resultados entre a troca valvar convencional, com a excisão 
FANTINI, F.A.; DRUMOND, L. F.; GONTIJO FILHO, B.; VRANDECIC, M. O.; PAULA E SILVA, J. A.; BARBOSA, J. T.; BRACARENSE, L. F.; OLIVEIRA, C. A. H.; GUTIERREZ, C.; PEDROZA, A. A.; HORTA, M. G. C. - Valvoplastia mitral em pacientes jovens com cardiopatia reumática. Rev. Bras. Cir. Cardiovasc., 7(2):78-87,1992.

completa da valva nativa, e cirurgias que preservem o aparelho subvalvar mostram que a fração de ejeção é conservada e que os índices de volumes sistólico e diastólico final do ventrículo esquerdo diminuem quando se mantêm intatos os elementos valvares ${ }^{8}$, 17, 22, 27. Além disso, segundo HENNEIN et alii ${ }^{27}$, isso melhora a sobrevida e aumenta a capacidade ao exercício a longo prazo.

No presente trabalho, a gravidade dos pacientes tratados pode ser comprovada pela grande proporção $(44,6 \%)$ de pacientes em classe funcional IV da NYHA. Além disso, 4 pacientes tiveram de ser operados em fase ativa da doença reumática, devido a grave instabilidade hemodinâmica. Apesar disso, observamos um baixo índice de complicaçōes no pós-operatório e uma progressiva melhora da funçăo ventricular. Assim, com a experiência acumulada e os resultados obtidos, temos procurado indicar o tratamento cirúrgico mais precocemente, baseados, principalmente, em critérios ecocardiográficos ${ }^{11}$.

Outra vantagem da valvoplastia, descrita na maioria das publicaçōes ${ }^{2}, 9,18,32$, é a menor incidência de trombo-embolismo e de endocardite. Na presente série, nāo foram observados casos destas complicações. No entanto, temos que considerar que, devido, provavelmente, à baixa média de idade dos nossos pacientes, no pré-operatório, somente 4 $(7,4 \%)$ apresentavam fibrilação atrial. No seguimento tardio, dois pacientes continuaram a apresentar essa arritmia, enquanto que um retornou a ritmo sinusal e outro nāo teve seguimento.

Um dado importante na análise dos resultados da valvoplastia relaciona-se à própria evoluçāo da doença reumática. Para AUTUNES et alii ${ }^{2}$ a cardite reumática persistente ou reativada pode ser considerada um significante fator de falha na correção. Tem importância fundamental a observação de que a doença reumática parece ter um caráter autoimune. Segundo ARGUELLES ${ }^{3}$, na fase aguda a valva seria atingida por anticorpos primariamente desenvolvidos contra o estreptococo. Na fase crônica, a valva lesada seria a responsável pela estimulação antigênica, levando à produção de anticorpos capazes de reagir contra ela própria. Títulos desse anticorpo poderiam sofrer rápidas e desproporcionais elevaçōes no decorrer de estímulos antigênicos, mesmo discretos, levando à recorrência da inflamaçāo, ou ao progressivo e subclínico agravamento da lesão valvar, mesmo em pacientes sem surto de estreptococcia ${ }^{5,6}$.

Desta forma, os pacientes reumáticos submetidos a cirurgias conservadoras continuam sujeitos à influência da doença e de outros fatores, como o stress mecânico produzido pela passagem do sangue por valvas deformadas e pela correçāo realiza- da, tornando o resultado a longo prazo incerto e aumentando o índice de reoperaçōes. $\mathrm{Na}$ nossa casuística, a recidiva da doença reumática foi, comprovadamente, a responsável por três das quatro reoperaçōes. A impossibilidade de se pesquisar a prevalência de recidivas em todos os casos em seguimento nos impossibilitou de verificar a significância estatística dessa observaçāo, muito embora outros relatos de recidivas, amigdalites e uso irregular de penicilina tenham sido coletados. Conforme demonstrado no Gráfico 5 , que compara os estudos ecocardiográficos do pós-operatório com os do período de acompanhamento, houve em alguns casos progressāo do grau de insuficiência mitral, mantendo-se, porém, estável na maioria.

Assim, nossos dados sugerem que o rígido controle da doença reumática tem importância fundamental no resultado a longo prazo, principalmente em pacientes mais susceptíveis a recidivas. Além disso, as observaçōes de AYOUB et alii ${ }^{6}$ de que ocorre uma redução de freqüência ou, mesmo, ausência de recorrência das crises de cardite quando se retira a valva doente, sāo forte incentivo para as pesquisas ora em desenvolvimento à procura de substitutos valvares mais duradouros e menos sujeitos a complicaçōes ${ }^{36}$

Além disso, inúmeros autores ${ }^{2,14,18,19}$ têm demonstrado que os resultados da valvoplastia mitral na doença reumática sāo inferiores, quando comparados a outras etiologias. As severas alteraçōes patológicas que, geralmente, acompanham a valvoplastia reumática já seriam obstáculo à realização da valvoplastia. De acordo com COSGROVE \& STEWART ${ }^{15}$, a realização de uma valvoplastia é possível em $75 \%$ dos casos, quando a etiologia é degenerativa e em apenas $49 \%$ quando é reumática. BONCHEK ${ }^{7}$ limita as valvoplastias a pacientes nāo reumáticos, enquanto GREGORI Jr. et alii ${ }^{24}$ relatam melhores resultados em valvas sem estenose associada.

Outro ponto importante a ser discutido diz respeito à técnica cirúrgica utilizada pelo nosso grupo desde o início da experiência. Vários aspectos foram considerados. No que diz respeito à alteração básica da insuficiência mitral reumática crônica, esta relaciona-se a uma retraçāo fibrótica das cúspides valvares, principalmente da posterior, levando a uma falha de coaptação e conseqüente dilatação do ventrículo esquerdo e anel valvar mitral, pela sobrecarga volumétrica ${ }^{4}$. Embora fatores, como a retraçāo ou alongamento das cordas estejam envolvidos, o que ocorre é uma escassez de tecido, e qualquer técnica cirúrgica utilizada induz a uma reduçāo do orifício valvar mitral. Outra consideraçāo relacionase ao emprego de anéis "fechados" para a valvoplastia, que restringe o crescimento do anel valvar, fato de particular importância em pacientes 
FANTINI, F.A.; DRUMOND, L. F.; GONTIJO FILHO, B.; VRANDECIC, M. O.; PAULA E SILVA, J. A.; BARBOSA, J. T.; BRACARENSE, L. F.; OLIVEIRA, C. A. H.; GUTIERREZ, C.; PEDROZA, A. A.; HORTA, M. G. C. - Valvoplastia mitral em pacientes jovens com cardiopatia reumática. Rev. Bras. Cir. Cardiovasc., 7(2):78-87,1992.

jovens, como os da presente série. $O$ uso de anéis "abertos", como o desenvolvido por GREGORI Jr. et alii ${ }^{23}$ resolve, em parte, esse problema. $O$ emprego de fitas de pericárdio como preconizado por JATENE* e, posteriormente, padronizado por BRAILE et alii ' nos parece mais adequado, por adaptar-se melhor ao contorno anular, além de não alterar a dinâmica do anel durante o ciclo cardíaco. Acreditamos que o uso desse mecanismo de contenção de uma possível nova dilatação do anel seja importante, já que ANTUNES \& KINSLEY ', em 1983, creditam a este fato parte das falhas ocorridas nas anuloplastias que realizaram.

Ainda tiveram importância na escolha da técnica os excelentes resultados apresentados por KALIL et alii **, em 1986, com índices de mortalidade hospitalar de $1,9 \%$ e de reoperaçāo de 10 anos de $5,8 \%$. Além disso, SAND et alii ${ }^{32}$ não consideraram

* Comunicação pessoal

** Trabalho apresentado no XUII Congresso Brasileiro de Cardiologia, Belo Horizonte, 1986. o tipo de anuloplastia usado como determinante dos bons resultados. Assim, resolvemos padronizar a técnica de anuloplastia preconizada por REED et alii ${ }^{31}$, complementando-a com outros procedimentos sobre as cúspides e aparelho subvalvar desenvolvidos por CARPENTIER et alii ${ }^{12}$.

Embora a nossa série careça de maior período de observação, podemos concluir que:

1) a valvoplastia mitral continua sendo a melhor opção no tratamento da insuficiência mitral reumática em pacientes jovens:

2) a cirurgia pode ser indicada mais precocemente e baseada em critérios ecodopplercardiográficos;

3) as técnicas de anuloplastia com o uso de pericárdio para a estabilização do anel valvar são excelente opção, principalmente no grupo de pacientes em idade de crescimento;

4) o controle clínico rígido, inclusive da estreptococcia, é fundamental na obtenção de bons resultados a longo prazo.

\section{RBCCV 44205-165}

FANTINI, F. A.; DRUMOND, L. F.; GONTIJO FILHO, B.; VRANDECIC, M. O.; PAULA E SILVA, J. A.; BARBOSA, J. T.; BRACARENSE, L. F.; OLIVEIRA, C.A.H.; GUTIERREZ, C.; PEDROSA, A.A.; HORTA, M.G.C. - Mitral valvuloplasty in young patients with rheumaticheart disease. Rev. Bras. Cir. Cardiovasc., 7(2):78-87,1992.

ABSTRACT: From September 1988 to July 1990,56 patients under 20 years of age (mean $12.7 \pm 5.12$ years) with pure or predominant mitral valve regurgitation secondary to rheumatic valve disease, underwent mitral valvuloplasty. Ninety-three percent of the patients were in functional class III ou IV (NYHA). The basic surgical technique used in all patients was a modified measured assymetric anuloplasty which was associated in $69.7 \%$ of the cases with another plastic procedure. Intraoperative echodopplercardiography was always used and showed a good correlation with the postoperatoty echo studies, with $64 \%$ of the patients free from residual lesions. There was no hospitalar mortality. Fifty-three $(94.6 \%)$ patients were followed from 1 to 40 months (mean $16.3 \%$ ). There was one sudden death 3 months after the operation. Four patients were reoperated upon, 3 of whom due to recurrent rheumatic carditis. The remainder are in functional class I or II (NYHA). We conclude based on these early results that assymetric mitral anuloplasty is an excellent procedure for young patients with rheumatic heart disease, being a good alternative to valve replacement or ring implantations.

DESCRIPTORS: mitral valve, surgery; rheumatic heart disease, mitral valvuloplasty; mitral valve, rheumatic heart disease.

AGRADECIMENTO: Agradecemos ao Dr. Dilermando Fazito, pela colaboração na análise estatística.

\section{REFERÊNCIAS BIBLIOGRÁFICAS}

valvuloplasty: results in an underdeveloped population. Thorax, 38: 730-736, 1983.

2 ANTUNES, M. J.; MAGALHĀES, M. P.; COLSEN, P. R.; KINSLEY, R. H. - Valvuloplasty for rheumatic mitral valve disease: a surgical challenge. $J$. Thorac. Cardiovasc. Surg., 94: 44-56, 1987.

3 ARGUELLES, E. - Anticorpo antiendocárdio e inversāo 
FANTINI, F.A.; DRUMOND, L. F.; GONTIJO FILHO, B.; VRANDECIC, M. O.; PAULA E SILVA, J. A.; BARBOSA, J. T.; BRACARENSE, L. F.; OLIVEIRA, C. A. H.; GUTIERREZ, C.; PEDROZA, A. A.; HORTA, M. G. C. - Valvoplastia mitral em pacientes jovens com cardiopatia reumática. Rev. Bras. Cir. Cardiovasc., 7(2):78-87,1992.

de mecanismo de auto-imunidade na fase crônica da febre reumática. Arq. Bras. Cardiol., 54:343346, 1990.

4 ARGUELLES, E. - Insuficiência mitral. In: ARGUELLES, E.; FISZMAN, P.; FAKOURY, L. (eds.) Febre reumática e doenças valvulares do coração. 2.ed. Rio de Janeiro, Revinter, 1984. p.309-415.

5 ASSIS, R. V. C.; GRINBERG, M.; LOPES, E. A.; DECOURT, L. V.; CORBETT, C. E. P.; PILEGGI, F.; JATENE, A. D. - Amiloidose e inflamaçāo em valvas mitrais calcificadas de pacientes reumáticos crônicos. Arq. Bras. Cardiol., 51: 433-436, 1988.

6 AYOUB, E. M.; TARANTA, A.; BASTLEY, T. D. - Effect of valvular surgery on antibody to the group $A$ streptococcal carbohydrate. Circulation, 50: 144-150, 1974.

7 BONCHEK, L., I. - Comentário In: SPENCER, F. C. COLVIN, S. B.; CULLIFORD, A. T. Experiences with the Carpentier techniques of mitral valve reconstruction in 103 patients $(1980-1985)$. J. Thorac. Cardiovasc. Surg., 90: 341-350, 1985.

8 BONCHEK, L. I.; OLINGER, G. N.; SIEGEL, R.; TRESCH, D. D.; KEELAN, M. H. - Left ventricular performance after mitral reconstruction for mitral regurgitation. J. Thorac. Cardiovasc. Surg., 88: 122-127, 1984

9 BRAILE, D. M.; ARDITO, R. V.; PINTO, G. H.; SANTOS, J. L. V.; ZAIANTCHICK, M.; SOUZA, D. R. S.; THEVENARD, R. - Plástica mitral. Rev. Bras. Cir. Cardiovasc., 5: 86-98, 1990.

BROFMAN, P.R. - Cirurgia conservadora da valva mitral em pacientes jovens: técnica cirúrgica e resultados. São Paulo, 1986. [Tese. Doutorado. Faculdade de Medicina da Universidade de São Paulo].

17 valvular regurgitation. Ann. Thorac. Surg., 53: 191199, 1992.

CARPENTIER, A. - Cardiac valve surgery: the "french correction". J. Thorac. Cardiovasc. Surg., 86: 323337,1983

CHARLESWORTH, D. C.; WEISEL, R. D.; BAIARD, R. J. - Assessment of mitral and tricuspide competence after valvuloplasty. Ann. Thorac. Surg., 35: 105$107,1983$.

CHAUVAUD, S.; PERIER,,P.; TOUATI, G.; RELLAND, J.; KARA, S.M.; BENOMAR, M.; CARPENTIER, A. - Longterm results of valve repair in children with acquired mitral valve incopetence. Circulation, 74 (Supl. 1): 104-109, 1986.

COSGROVE, D. M. \& SREWART, W. J. - Mitral valvuloplasty. Curr. Probl. Cardiol., 14: 359-415, 1989.

COUTO, A.A.; CARNEIRO, R.D.; GIRARDI, J.M.; OLI-

VEIRA, G.M.M. - Estado atual da patogenia da doença reumática. Arq. Bras. Cardiol., 50: 75 $78,1988$.

DAVID, T. E.; UDIÉN, D. E.; STRAUSS, H. D. - The importance of the mitral apparatus in left ventricular function after correction of mitral regurgitation. Circulation, 68(Supl. 2): 76-81, 1983.

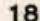

DELOCHE, A.; JEBARA, V. A.; RELLAND, J. Y. M.; CHAVAUD, S.; FABIANI, J. N.; PERIER, P.; DREYFUS. G.; MIHAILEANU, S.; CARPENTIER, A. - Valve repair with Carpentier techniques: the second decade. J. Thorac. Cardiovasc. Surg. 99: 990$1002,1990$.

DURAN, C. G.; REVUELTA, J. M.; GAITE, L.; ALONSO, C.; FLEITAS, M. G. - Stability of mitral reconstructive surgery at 10-12 years for predominantly rheumatic valvular disease. Circulation, 78(Supl. 1): 91-96, 1988.

ELOY, R.; FERREIRA Jr., A.; CARVALHO, H.; BRITO, J. C.; TADEU, E; NERY, A. C.; RIBEIRO, A.; OLIVEIRA, R.; RIBEIRO, N. - Cirurgia orovalvar na criança: experiência de 135 casos. Rev. Bras. Cir. Cardiovasc., 1:24-28, 1986.

GALLOWAY, A. C.; COLVIN, S. B.; BRAUMANN, G.; ESPOSITO, R.; VOHRA, R.; HARTY, S.; FREEDBERG, R.; KRONZON, I.; SPENCER, F. Longterm results of mitral valve reconstruction with Carpentier techniques in 148 patients with mitral insufficiency. Circulation, 78(Supl. 1): 97-105, 1988.

GOLDMAN, M. E.; MORA, F.; GUARINO, T.; FUSTER, V.; MINDICH, B. P. - Mitral valvuloplasty is superior to valve replacement for preservation of left ventricular function: an intraoperative two-dimensional echocardiographic study. J. Am. Coll. Cardiol., 10: 568-575, 1987.

GREGORI Jr., F.; SILVA, S. S.; BABA, K.; QUEIROZ, L. T.; TAKEDA, R.; FAÇANHA, L. A.; SHIGUERU, S.; CANESIN, O. - Um novo modelo de anel protético para pacientes com insuficiência valvar mitral: relato de 2 casos. Arq. Bras. Cardiol., 50:417-420, 1988 .

GREGORI Jr., F.; SILVA, S.S.; MOURE, O.; TAKEDA, R.; FAÇANHA, L.; RIBEIRO, I.; GÓIS, L.; SIQUEIRA, J.; GREGORI, T. F.; LOPES, A. G.; KRELING, P.; MIGUITA, L. C.; CORDEIRO, C. - Tratamento cirúrgico da insuficiência mitral em crianças: dez anos de técnicas reparadoras. Rev. Bras. Cir. Cardiovasc., 4: 202-209, 1989.

GRUNKEMEIER, G. L. - Statistical analysis of prosthetic valve series. In: RABAGO, G. \& COOLEY, D. A. (eds.) Heart valve replacement: current status and future trends. Mount Kisco, Futura Publishing, 1987. p.11-25. DERBY, G. C.; CAHILL, P. D.; MILLER, D.C. - 
FANTINI, F.A.; DRUMOND, L. F.; GONTIJO FILHO, B.; VRANDECIC, M. O.; PAULA E SILVA, J. A.; BARBOSA, J. T.; BRÁCARENSE, L. F.; OLIVEIRA, C. A. H.; GUTIERREZ, C.; PEDROZA, A. A.; HORTA, M. G. C. - Valvoplastia mitral em pacientes jovens com cardiopatia reumática. Rev. Bras. Cir. Cardiovasc., 7(2):78-87,1992.

Physiologic role of the mitral apparatus in left ventricular regional mechanics, contraction synergy and global systolic performance. J. Thorac. Cardiovasc. Surg., 97: 521-533, 1989.

HENNEIN, H. A.; SWAIN, J. A.; McINTOSH, C. L.; BONOW, R. O.; STONE, C. D.; CLARK, R. E. Comparative assessment of chordal preservation versus chordal resection during mitral valve replacement. J. Thorac. Cardiovasc. Surg., 99: 828-837, 1990.

KALIL, R. A. K.; PRATES, P. R.; LUCCHESE, F. A.; BERTOLETTI, V. E.; PEREIRA, E. M.; SANT' ANNA, J. R. M.; NESRALLA, I. A. - Valvuloplastia para insuficiência mitral: técnicas e resultados. Arq. Bras. Cardiol., 37 : 315-319, 1981.

LESSANA, A.; VIET, T. T.; ADES, F.; KARA, S. M.; AMEUR, A.; RUFFENACH, A.; GUERIN, F.; HERREMAN, F.; DEGEORGES, M. - Mitral reconstrutive operations: a series of 130 consecutive cases. J. Thorac. Cardiovasc. Surg., 86: $553-$ $561,1983$.

MANSNER, J. S. \& KRAMER, S. - Epidemiology: an introductory text. Philadelphia, W. B. Saunders, 1985. p. 329-353.

REED, G. E.; TICE, D. A.; CLAUSS, R. H. - Asymetric exaggerated mitral annuloplasty: repair of mitral insufficiency with hemodynamic predictability. J. Thorac. Cardiovasc. Surg., 49: 752-761, 1965.

SAND, M. E.; NAFTEL, D. C.; BLACKSTONE, E. H.; KIRKLIN, J. W.; KARP, R. B. - A comparison of repair and replacement for mitral valve incompetence. J. Thorac. Cardiovasc. Surg., 94: 208219, 1987.

SARRIS, G. E.; CAHILL, P. D.; HANSEN, D. E.; DERBY, G. C.; MILLER, D. C. - Restoration of left ventricular systolic performance after reattachment of mitral chordae tendineae: the importance of valvularventricular interaction. J. Thorac. Cardiovasc. Surg., 95: 969-979, 1988

SPENCER, F. C.; COLVIN, S. B.; CULLIFORD, A. T.; ISOM, O. W. - Experiences with the Carpentier techniques of mitral valve reconstruction in 103 patients (1980-1985). J. Thorac. Cardiovasc. Surg., 90:341-350, 1985.

TOMPKINS, D. G.; BOXERBAUM, B.; LIEBMAN, J. Longterm prognosis of rheumatic fever patients receiving regular intramuscular benzathine penicillin. Circulation, 45: 543-550, 1972.

VRANDECIC, M. O.; GONTIJO F', B.; OLIVEIRA, S. A.; SILVA, J. A. P.; FANTINI, F. A.; BARBOSA, J. P.; AVELAR, S. O. S.; PAWLOWSKI, A.; ANDRADE, C. A. O.; MIOTTO, H. C.; BRAGA, M. A.; GUTIERREZ, C. - Tratamento anticalcificante de bioprótese: resultado clínico inicial. Rev. Bras. Cir. Cardiovasc. (No prelo).

\section{Discussão}

DR. LUIZ ANTÔNIO TENO

Ribeirāo Preto, SP.

Agradeço inicialmente à Comissāo do Congresso, pelo honroso convite de comentar o trabalho apresentado pelo $\mathrm{Dr}$. Fantini e colaboradores. O comprometimento de vários componentes do aparelho valvar na doença reumática é o que limita os resultados na plastia mitral, quando comparados com resultados dos procedimentos em outra etiologia. Apesar dos rigores dispensados à seleção dos casos, nos deparamos, em algumas situaçōes, com a impossibilidade de preservar a valva. No trabalho apresentado, essa situação ocorreu em apenas 3 casos, o que dá um índice de aproveitamento $(95 \%)$ considerado excelente quando comparado à literatura. Em série publicada recentemente por Duran e colaboradores, num total de 203 pacientes previamente selecionados para a cirurgia conservadora, 49 casos $(24,1 \%)$ tiveram o procedimento mudado para prótese mitral apenas na inspeção da valva no ato operatório; em 18 casos $(8,9 \%)$ foi tentada plastia, inicialmente, porém considerada nāo satisfatória pelo refluxo residual e substituída por prótese mitral no mesmo ato operatório. Apenas os restantes 136 casos foram submetidos a plastia conservadora e considerados como resultado satisfatórios, significando um índice de aproveitamento de $67 \%$ dos casos pré selecionados. Dados semelhantes de literatura também podem ser observados na série publicada por Deloche et alii, em 1990, e por Loop e Cosgrove, mais recentemente, em 1991, cujo índice de conservação da valva na doença reumática foi de $70 \%$. Gostaria de saber do Dr. Fantini a que fatores ele atribuiu o maior índice de conservação da valva na sua série, e se os casos de insuficiência mitral moderada $(3,6 \%)$ relatados na alta hospitalar não deveriam ter sido reconhecidos como tentativa sem sucesso e optado por colocaçāo de prótese no mesmo ato cirúrgico. Quanto às técnicas cirúrgicas utilizadas, em que predominam a anuloplastia assimétrica de REED associada à fixação do hemianel posterior com fita de pericárdio, a experiência dos autores com a mesma justifica a sua utilização preferencial. Gostaria também de fazer algumas consideraçōes em relação aos pacientes que foram submetidos a cirurgia na vigência da atividade reumática e os índices de reoperação encontrados na série apresentada. Dos fatores que contribuem para a falência cardíaca e instabilidade hemodinâmica na doença reumática na fase ativa, a maioria deles se deve à miocardite aguda que se instala. E acredita-se que, nessa fase, o comprometimento mecânico atribuído à disfunção valvar é responsável apenas parcialmente pelo estado geral agravado em que se encontram esses pacientes. $E$ a sua abordagem deve 
FANTINI, F.A.; DRUMOND, L. F.; GONTIJO FILHO, B.; VRANDECIC, M. O.; PAULA E SILVA, J. A.; BARBOSA, J. T.; BRACARENSE, L. F.; OLIVEIRA, C. A. H.; GUTIERREZ, C.; PEDROZA, A. A.; HORTA, M. G. C. - Valvoplastia mitral em pacientes jovens com cardiopatia reumática. Rev. Bras. Cir. Cardiovasc., 7(2):78-87,1992.

ser diferente da encontrada em outras situaçōes, como a que ocorre na endocardite infecciosa com má evolução clínica, em que o comprometimento valvar é significativamente importante e responsável pela instabilidade hemodinâmica. Na nossa experiência, no Instituto do Coração da Sta. Casa de Ribeirăo Preto, năo temos nenhum caso que tenha sido encaminhado para correçăo cirúrgica conservadora da valva mitral, numa fase sabidamente reconhecida como de atividade reumática. Nós temos, ao contrário, casos de insuficiência mitral em pacientes jovens em fase ativa da doença, que tiveram uma área cardíaca reduzida e, praticamente, desaparecimento ou insuficiência residual mínima após terapêutica clínica agressiva. Essa observaçāo foi também feita por Duran e colaboradores, que, em sua série, todos os pacientes que foram operados em atividade reumática foram reoperados para colocação de prótese mitral. E na seqüência, concluíram que: "No momento, considera-se que não deveriam ser tentadas as plastias na valva mitral na presença de atividade reumática". Em relaçāo ao índice de reoperaçāo apresentado na sua série, quando comparado com outras séries de literatura, ele se apresenta bastante satisfatório. Eu gostaria de saber da sua opiniāo se esse índice não pode estar mascarado pelo curto tempo de acompanhamento e se os casos operados na fase ativa năo poderāo vir a comprometer esses resultados. Também gostaria de perguntar ao Dr. Fantini, com referência à sua conclusāo de que a cirurgia pode ser indicada mais precocemente, se os critérios de indicaçăo cirúrgica da insuficiência mitral no jovem permanecem os mesmos, ou deveriam ser menos rigorosos? Encerrando, quero parabenziar os autores e salientar que trabalhos de importância e excelente qualidade como este apresentado pelo $\mathrm{Dr}$. Fantini e colaboradores é que trarāo luzes a essas consideraçōes que ainda nos inquietam, pois devemos lembrar que a reconstruçăo cirúrgica da valva mitral em jovens não é totalmente isenta de complicaçōes, principalmente as de natureza técnica, cuja necessidade de utilização de prótese num acompanhamento a curto prazo é sempre uma possibilidade a ser considerada. Muito obrigado.

\section{DR. FANTINI}

\section{(Encerramento)}

Primeiramente, gostaria de agradecer ao $\mathrm{Dr}$. Luiz Antonio, principalmente pelo sentido crítico com que analisou os nossos dados, levantando dúvidas pertinentes e contribuindo sobremaneira para o enriquecimento do tema. Quanto aos fatores que contribuíram para o alto índice de conservaçăo das valvas nativas, na presente série, cremos que sāo três: primeiramente, os critérios ecocardiográficos extremamente rígidos que temos utilizado na seleçăo dos pacientes; em segundo lugar, a baixa faixa etária dos pacientes, portanto com valvas menos deformadas e que possibilitam aos ecocardiografistas melhor observaçāo dos detalhes, e, por último, a tenacidade dos nossos cirurgiōes em realizar o procedimento. Os casos que apresentavam insuficiência mitral moderada na ocasião de alta hospitalar a princípio nos preocuparam e poderiam ter sido revistos caso o eco per-operatório tivesse mostrado esse grau de regurgitaçăo. Porém, como pôde ser visto na apresentaçāo, a evoluçăo posterior, nos surpreendeu, visto que em um caso a lesāo residual não tem progredido e no outro, em decorrência de recidiva da doença reumática, houve progressão para uma estenose severa da valva, o que propiciou uma das reoperaçōes. A indicação cirúrgica na vigência de doença reumática também não é a rotina do nosso serviço. No entanto, os pacientes operados nessa fase foram recebidos em situação crítica, sem resposta adequada ao tratamento clínico inicial, sendo a insuficiência mitral considerada importante na instabilidade hemodinâmica. A evoluçāo pós-operatória comprovou essa possibilidade, já que esses pacientes evoluíram satisfatoriamente no pósoperatório imediato. Apenas um evoluiu, posteriormente, para troca mitral, provavelmente muito mais em decorrência de problemas sócio-econômicos, já que nāo fez uso da medicaçāo recomendada. Os demais encontram-se bem. A indicação de troca valvar nesses casos, como proposto por Duran, é, ao nosso ver, polêmica, já que Antunes, em 1987, mostrou bons resultados numa larga série de pacientes operados na fase ativa da doença. Quanto à indicaçăo cirúrgica mais precoce, temos observado, baseados em estudos ecocardiográficos seriados, que a classe funcional é, em alguns casos, um critério falho. Assim, acreditamos que os bons resultados apresentados, aliados ao alto índice de preservaçāo das valvas, justificam uma indicaçāo mais precoce, antes que a insuficiência crônica leve a uma deterioraçāo ventricular esquerda que possa prejudicar o resultado a longo prazo. Concordamos que o período de observaçāo é ainda curto, e o tempo certamente trará as respostas necessárias. No entanto, năo podemos nos esquecer que, antes de estarmos simplesmente corrigindo valvas defeituosas, estamos, na realidade, atuando numa entidade complexa, de difícil tratamento e com profundas implicaçōes sócio-econômicas e culturais. 